\title{
A Computer Simulation of the System-Wide Effects of Parallel-Offset Route Maneuvers
}

\author{
Todd A. Lauderdale* \\ NASA Ames Research Center, Moffett Field, CA, 94035 \\ Confesor Santiago ${ }^{\dagger}$ \\ Federal Aviation Administration, William J. Hughes Techincal Center, Atlantic City, NJ, 08405 \\ Carl Pankok \\ General Dynamics Information Technology, Mays Landing, NJ, 08330
}

\begin{abstract}
Most aircraft managed by air-traffic controllers in the National Airspace System are capable of flying parallel-offset routes. This paper presents the results of two related studies on the effects of increased use of offset routes as a conflict resolution maneuver. The first study analyzes offset routes in the context of all standard resolution types which air-traffic controllers currently use. This study shows that by utilizing parallel-offset route maneuvers, significant system-wide savings in delay due to conflict resolution of up to $30 \%$ are possible. It also shows that most offset resolutions replace horizontal-vectoring resolutions. The second study builds on the results of the first and directly compares offset resolutions and standard horizontal-vectoring maneuvers to determine that in-trail conflicts are often more efficiently resolved by offset maneuvers.
\end{abstract}

\section{Introduction}

ODERNIZATION of the current air transportation system is an ongoing, incremental process. An early

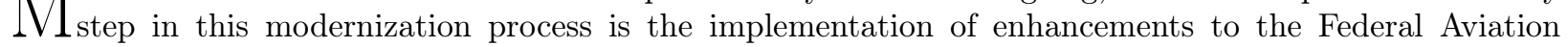
Administration's (FAA's) En Route Automation Modernization (ERAM) that supports en-route controllers in managing separation. This project is called Trajectory Based Operations (TBO) Separation Management: Modern Procedures. Research supporting this project involves computer human interface improvements to ERAM's Decision Support Tools (DSTs) post Release 3 and algorithmic enhancements to the Conflict Probe to increase the usability of the tools for the near-term time frame of NextGen. One proposed algorithmic enhancement is to enable ERAM to model parallel-offset routes in its trajectory predictions ${ }^{1}$ (Figure 1). This enhancement would allow air-traffic controllers to use parallel-offset routes as solutions to predicted separation violations, taking advantage of aircraft Flight Management Systems (FMS) capabilities.

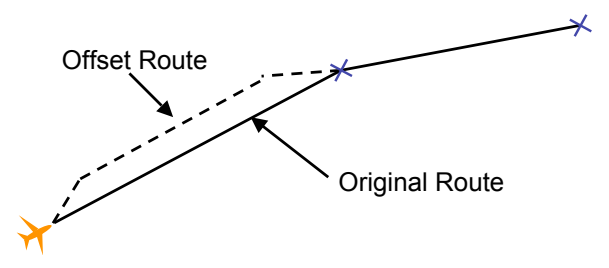

Figure 1. A schematic of a parallel-offset route maneuver.

There are many possible benefits of incorporating parallel-offset routes into air-traffic-management operations. Use of these types of maneuvers for conflict resolution may increase resolution efficiency by sup-

*Aerospace Engineer, NASA Ames Research Center, AIAA Member

${ }^{\dagger}$ Computer Scientist, FAA Simulation and Analysis Team, AJP-661, AIAA Member

$\ddagger$ Operations Research Analyst, General Dynamics Information Technology 
plementing the current standard resolutions, such as altitude clearances and horizontal vectoring. Offset routes are also closed trajectories that are theoretically easy to specify to a pilot via voice communication, and they are a standard, easily implemented feature of most modern FMS systems. Only a turn-out angle, an offset distance, and a return way point are required to specify a full, closed trajectory. Finally, since these trajectories are closed, accurate aircraft intent is available to ground-based decision-support tools and conflict probes, such as Conflict Alert, providing a benefit in terms of conflict prediction accuracy. ${ }^{2,3}$

The purpose of this paper is to examine the conflict resolution efficiency aspects of supplementing current conflict resolution maneuvers with offset-route maneuvers. Two related studies will be presented. The first is a system simulation using a representation of an air-traffic controller to determine when offset resolutions are preferable to other types of resolutions and to determine what are the system-wide effects of these offsets. Specifically, it is important to understand whether greater utilization of offset routes will lead to an increase or decrease in the total number of conflicts and in the total system-wide delay due to conflict resolution. Also, individual cases where offset routes are more efficient than other resolution methods are identified to determine their properties. The second study is predicated on the results of the first experiment and explores more deeply the relative merits of horizontal-vectoring resolutions and offset resolutions. These studies are designed to inform the Separation Management project on the possible benefits of parallel-offset routes and to indicate candidate situations where they might be best utilized.

\section{Simulation Environment}

For the studies presented, fast-time simulations of the National Airspace System (NAS) are conducted. The Airspace Concept Evaluation System (ACES) ${ }^{4}$ is used to simulate the National Airspace System, and the Advanced Airspace Concept (AAC) Autoresolver ${ }^{5,6}$ is used to approximate the decisions of an airtraffic controller with regard to selection of different conflict resolution maneuvers. ACES is a gate-to-gate NAS-wide simulation platform with a four degree-of-freedom trajectory generator. Aircraft trajectories are created from the departure fix to the arrival fix with performance data derived from the Base of Aircraft Data (BADA). ACES includes the capability to model trajectory-prediction uncertainty, but for the current studies, perfect trajectory prediction was used.

The AAC Autoresolver takes advantage of the trajectory capabilities of ACES to detect and resolve predicted losses of separation between aircraft as well as to space aircraft at metering fixes and avoid weather. The Autoresolver uses an iterative search over many different resolution types to select the most efficient resolution, based on minimum delay, for each airspace problem. The different types of resolutions attempted to resolve loss-of-separation conflicts included vertical resolutions such as step climbs or descents, horizontal resolutions such as path stretches, and speed resolutions.

For the studies in this paper, a parallel-offset resolution was added to the types of resolutions considered by the Autoresolver. These resolutions were available for all non-arrival aircraft that have a straight route for at least the next $100 \mathrm{nmi}$. The turn-out angle for offset resolutions was set at 30 degrees, and two offset distances were tried: $6 \mathrm{nmi}$ and $10 \mathrm{nmi}$. The aircraft stayed on the offset route for 50, 100 or $150 \mathrm{nmi}$ depending on if the return to the route was conflict-free.

\section{First Study: Air-Traffic Controller Approximation}

As discussed earlier, the transformation into NextGen is expected to occur incrementally with one of the first steps possibly including enhanced parallel-offset route capabilities. Theoretically, including parallel-

offset resolutions as a conflict resolution tool for air-traffic controllers will increase the possible options for resolution maneuvers and therefore improve system efficiency. The purpose of the first study presented here is to determine if this benefit is realizable using realistic and varied aircraft traffic and conflict scenarios. The effects of offset resolutions on the number of conflicts in the system and total system delay due to conflict resolutions was studied. In addition, particular conflict situations were analyzed to determine when offsets are particularly useful.

\section{A. Configuring the Autoresolver}

For this fast-time simulation, the AAC Autoresolver was used to approximate the actions of air-traffic controllers. To more closely approximate their decisions, parameters which modify the performance of the 
Autoresolver were selected so that the resulting resolutions more closely matched the properties of resolutions observed in the current system. Specifically, two parameters were adjusted in the Autoresolver: a bias towards horizontal resolutions and the efficiency of path-stretch resolutions.

The horizontal bias can be thought of as a time handicap for speed and vertical resolutions. If the horizontal bias is set to one minute, then for a vertical or speed resolution to be selected then it must have at least one minute less delay than the best horizontal resolution. Adjusting this parameter allowed for control over the proportion of each type of resolution selected by the Autoresolver

An analysis of current operations was performed to help calibrate this parameter. This analysis was an extension of the work by Paglione et al. ${ }^{7}$ which presented an algorithm to determine the number and geometric properties of conflicts in the current system. For this analysis, their algorithm was extended to determine the type of maneuvers used to resolve each conflict. Aggregating these numbers, the overall proportion of the conflicts in the current system which were resolved using horizontal, vertical or speed maneuvers were obtained.

Two six-hour periods of operation (April 3, 2008 and April 16, 2009) were analyzed. Figure 2(a) shows the conflict resolution distribution for all traffic in the system. The largest proportion of resolutions were horizontal resolutions followed by vertical resolutions. Speed resolutions accounted for approximately $14 \%$ of all resolutions. The conflict proportion can also be determined for individual Air Route Traffic Control Centers (Centers), and the results are shown in Figure 2(b) for a representative sample of centers: Boston (ZBW), Indianapolis (ZID), Minneapolis (ZMP), and Oakland (ZOA). Using these results, the horizontal bias was tuned to mimic this general behavior for each center.

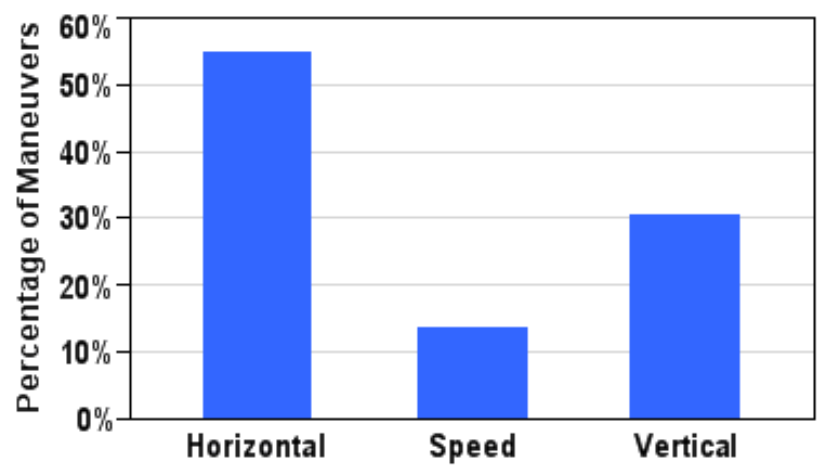

(a)

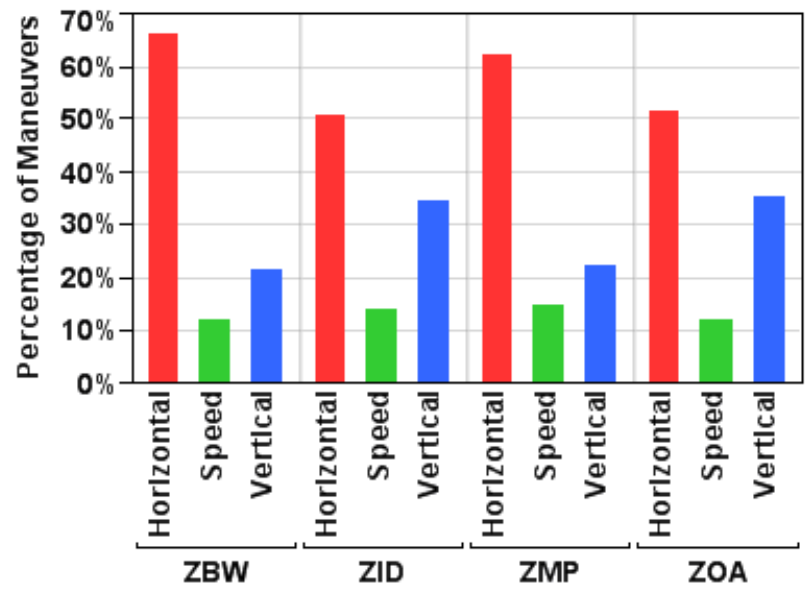

(b)

Figure 2. The (a) aggregate proportion of resolution types for four centers, and (b) the proportion of resolution types for individual centers. 
The second Autoresolver parameter set for these runs was the efficiency of the path-stretch maneuver. In current practice, horizontal-vector maneuvers are issued in a two-step open trajectory process. First, the aircraft is directed to turn to a new heading. The controller resumes scanning the airspace and then at a later point directs the maneuvered aircraft to return to its route. A path-stretch maneuver is a closed trajectory form of this maneuver used by the Autoresolver where a single auxiliary waypoint is added to an aircraft's route (Figure 3).

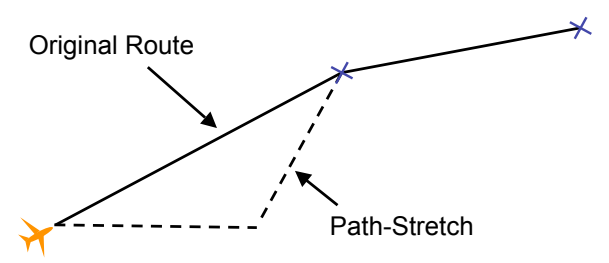

Figure 3. A schematic of a path-stretch maneuver.

To mimic the current horizontal-vectoring process using path stretches in the Autoresolver, an additional delay is added to the path stretch chosen by the Autoresolver. Based on consultation with subject matter experts at the FAA, a Gaussian distribution was selected for this additional delay with a mean of 0 seconds and a standard deviation of 20 seconds. Random samples were taken from this distribution and the absolute value of this number was taken to determine the desired extra delay for the horizontal vector. A new path stretch was then created with the same turn-out angle as the original path-stretch resolution but with a total delay increased by the extra delay value (Figure 4).

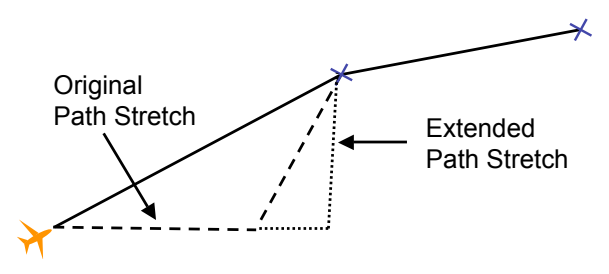

Figure 4. A schematic of an extended path-stretch resolution.

\section{B. Simulation Setup}

For this analysis, the effects of including parallel-offset routes were analyzed for the predicted demand in the years 2014, 2018 and 2025 using 24 hours of predicted traffic data for each. The Autoresolver was configured for each run to operate in one of the four Centers ZBW, ZID, ZME, and ZOA. For each Center and demand year, one run was used as a baseline where no parallel-offset routes were allowed, and a second run was performed in which parallel-offset routes were a candidate resolution maneuver. Between the centers, years, and baseline and offset cases there were a total of 24 ACES runs performed.

\section{System-Wide Results}

In the analysis of the efficiencies and benefits of using parallel-offset route resolutions to air-traffic separation violations, it was found that these types of maneuvers significantly reduced the number of conflicts, the amount of fuel consumption, and the delay. A detailed description of the benefits associated with including parallel offsets is given by Santiago and Paglione, ${ }^{8}$ but some top-level results from that study are presented here. In the following discussion, the results from each of the four Centers are aggregated and presented as a single result, but the detailed study ${ }^{8}$ includes a discussion of Center-level variations.

Figure 5(a) shows the aggregate number of conflicts for the four Centers in the experiment for the three demand levels. This figure shows that including offset-route resolutions in the system slightly reduces the total number of conflicts. There was also a reduction in conflict resolution related delay of up to $30 \%$ as shown in Figure 5(b). 


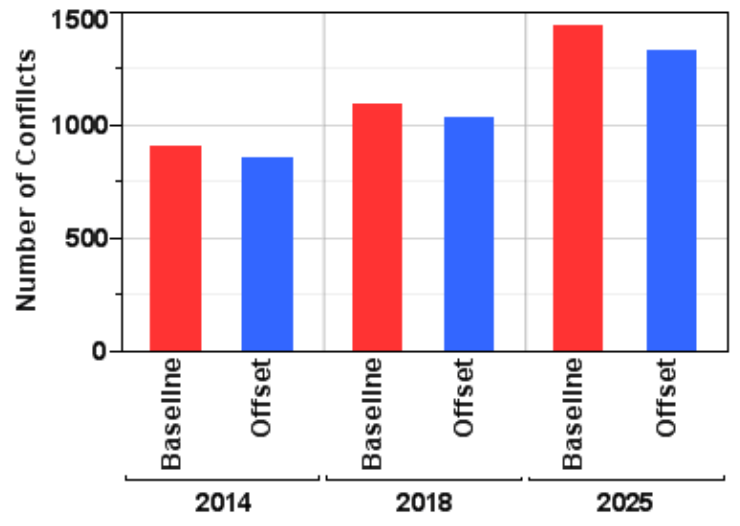

(a)

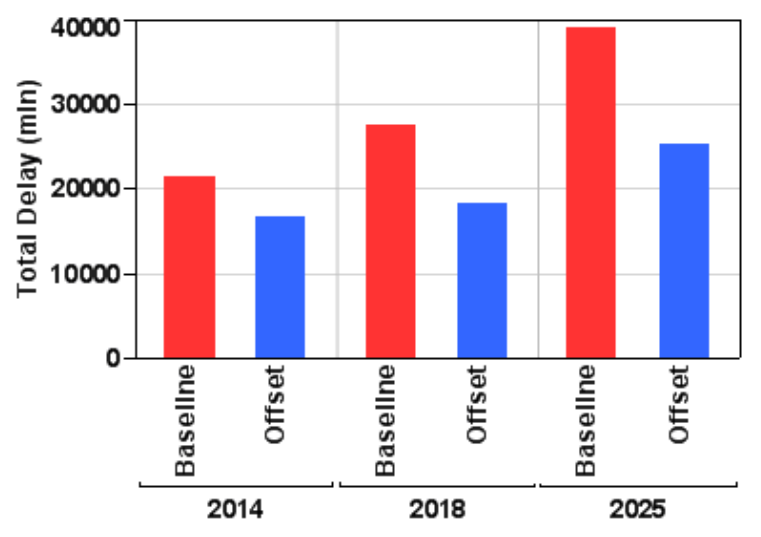

(b)

Figure 5. The (a) total number of conflicts and (b) the total delay for the baseline and offset cases for each demand set.

\section{Offset Conflict Properties}

While previous work focused on NAS-wide performance effects, this section highlights an investigation of the characteristics and trends of air-traffic separation and conflict properties during offset maneuvers. Relevant findings from this investigation will help feed into the development of requirements and validation of offset maneuvers. In addition, properties of these conflicts could help subject matter experts visualize and conceptualize the new automation procedure and understand specific types of situations where the procedure may be beneficial.

As noted before, offset conflict resolutions were only allowed in the offset runs. All other experiment settings remained constant. Figure 6(a) illustrates the distribution of conflict resolutions recorded in the baseline case, and Figure 6(b) shows this distribution for the offset case. As a general trend, there is a noticeable decrease in the percentage of path-stretch maneuvers and vertical maneuvers when going from the baseline case to the offset case. This result suggests that, in general, parallel offset maneuvers are replacing path-stretch and vertical maneuvers more than they are replacing the other resolution maneuvers.

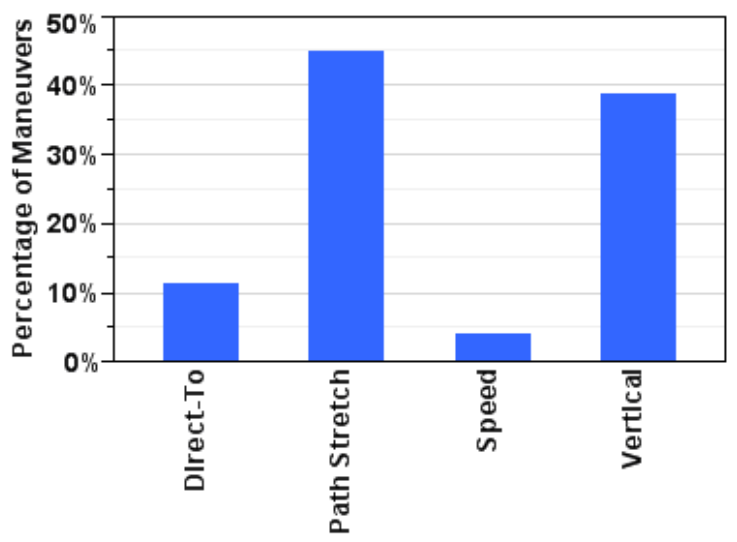

(a)

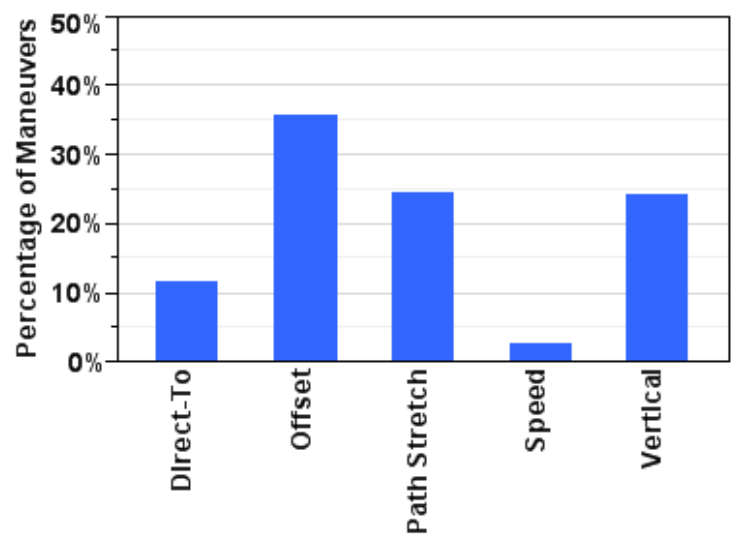

(b)

Figure 6. The overall distribution of conflict resolutions maneuvers for (a) the baseline case and (b) the offset case.

To further investigate these findings, conflicts in the baseline case which were also present in the offset case were identified. This set of resolutions allowed for an understanding of which types of maneuvers were being replaced by offset resolutions in the offset case. Figure 7 illustrates the distribution of replaced 
maneuvers. The figure reveals that path stretches are the most frequently replaced maneuver and vertical maneuvers are also replaced in significant quantities. Overall, $57 \%$ of the maneuvers replaced by an offset were path-stretch maneuvers. The high correlation of path-stretch and offset conflict resolutions shows that there is a unique relationship, which is understandable since both maneuvers lie in the lateral domain. This finding leads to the second experiment presented here to study sensitivities of the operational parameters the model uses to implement path stretches and offsets.

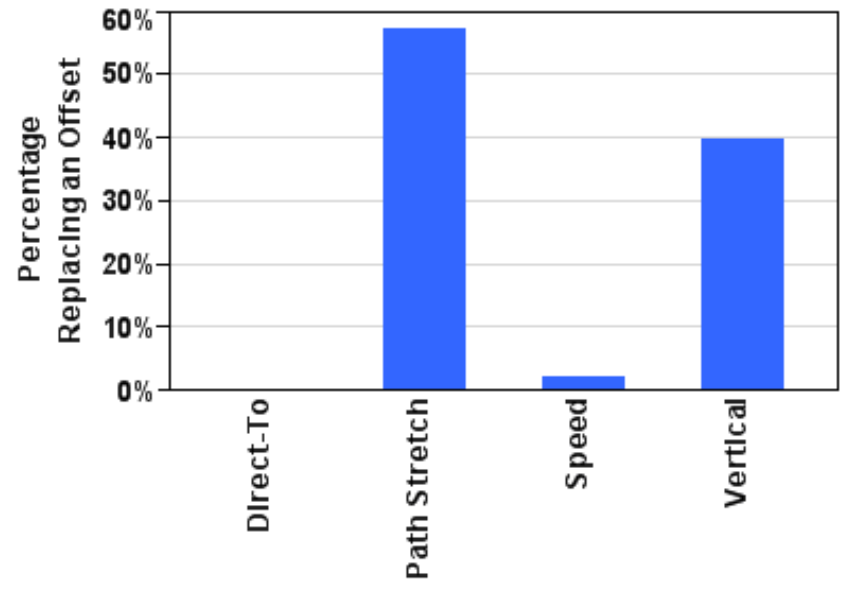

Figure 7. The percentage of each maneuver which was replaced by an offset maneuver.

\section{Second Study: Comparing Offsets and Horizontal Vectoring}

The first study presented in this paper demonstrated that, in terms of conflict resolution maneuvers, parallel-offset maneuvers are most closely related to path-stretch maneuvers. The purpose of this second study was to explore the relationship between these two types of maneuvers in greater detail by directly comparing offsets and path stretches for each resolution attempt independently of which resolution was actually selected for execution by the Autoresolver. As discussed before, a two-step horizontal-vectoring maneuver may not be as efficient as the corresponding path-stretch maneuver selected by the Autoresolver, so the results derived from this comparison were explored as a function of this path-stretch efficiency.

\section{A. Simulation Setup}

In order to determine how the results vary with path-stretch efficiency, a slightly different methodology for varying path-stretch efficiency was used for this study. As before, the most efficient path-stretch was determined by the Autoresolver. But, instead of sampling from a distribution to determine the additional delay, a fixed additional percentage of delay ranging from $0 \%$ to $100 \%$ was added to the optimal-delay resolution. So, at $0 \%$ the optimal path stretch was used by the Autoresolver. At $100 \%$, a resolution twice as long as the optimal resolution was created and used as the available path-stretch resolution. Varying this parameter allows for an understanding of how offsets compare even when path-stretch resolutions are completely efficient and when path-stretch resolutions are very inefficient. The actual operating point will most likely be somewhere between these two extremes.

For this study all 20 Centers are used with an independent Autoresolver agent in each Center. ${ }^{9}$ The additional path-stretch delay parameter is set to values of $0,25,50,75$, and 100 percent. Demand sets with predicted demand for the years 2014 and 2018 are sampled for the busiest three hours of a day (10:00 to 13:00 UTC). Each of these two different data sets are run for each delay parameter value.

\section{B. Results}

After completing the 10 runs for the different values of path-stretch delay and the different demand sets, the results were analyzed to directly compare only the path-stretch and offset results for each conflict. So, 
for each conflict, it was determined if there was at least one successful path-stretch resolution and at least one successful offset-route resolution. For the cases where there were successful resolutions of both types the most efficient resolution type was designated.

Figure 8 shows the percentage of all resolutions where there is a successful offset resolution which has less delay than the best available path-stretch resolution. Roughly $17 \%$ of the time that there is a conflict an offset resolution is more efficient than a path-stretch resolution. This percentage seems to vary approximately linearly with the path stretch efficiency parameter. The greater traffic density for the 2018 demand set causes a slight decrease in the percentage of offset resolutions which were more efficient than path-stretch resolutions possibly because there are more secondary conflicts with surrounding traffic.

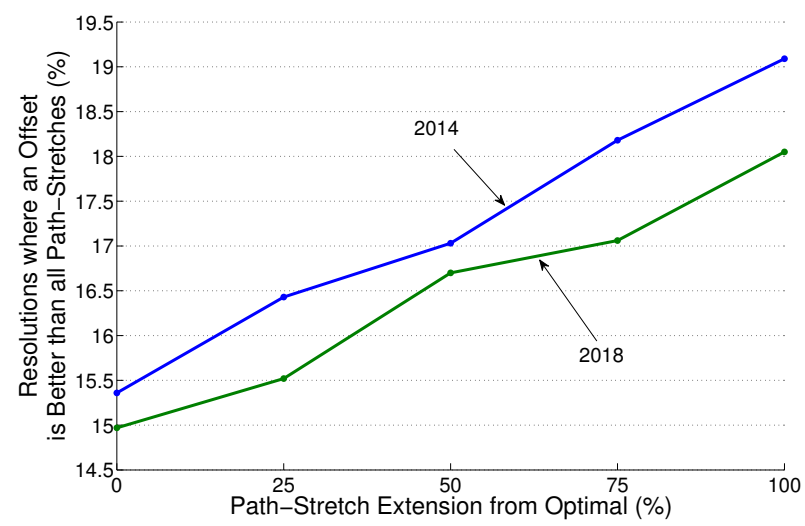

Figure 8. The percentage of all conflicts where a parallel-offset resolution is more efficient than the best path-stretch resolution found by the Autoresolver.

The average time savings for cases where an offset resolution was more efficient than the related path stretch are shown in Figure 9. In this figure, both types of resolution are successful for the same aircraft in the same conflict. The time savings when an offset was more efficient than a path stretch was on the order of 27 seconds per resolution for all cases and increased non-linearly as a function of the path stretch efficiency parameter. Greater savings were realized for the higher traffic densities of the 2018 demand set. These results are shown in more detail in a histogram in Figure 10 for the 2014 demand set and the 50\% path-stretch extension case. It can be seen that most of the cases have a savings of less than 50 seconds, but there are cases with over 200 seconds of savings.

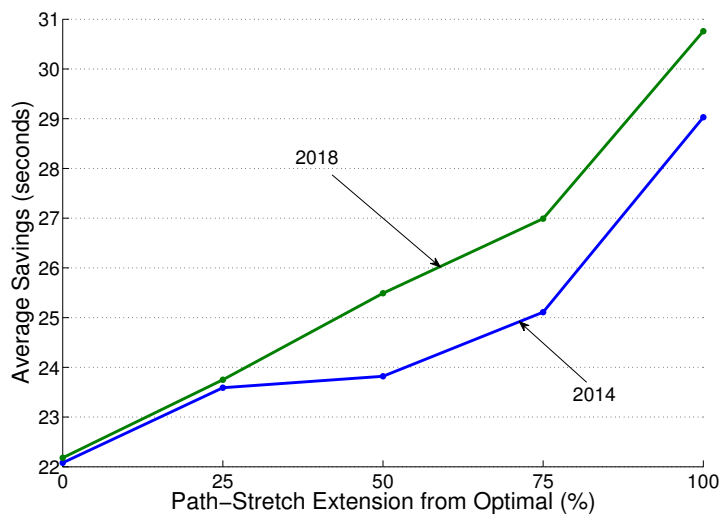

Figure 9. The average time savings of offset resolutions as compared to the best path-stretch resolution when both are available.

Figure 11 shows for three different conflict-angle bins what percentage of the resolutions in that bin are handled more efficiently with an offset resolution. The conflict angle is defined as the angle between the conflicting aircraft when the conflict is detected. An angle of 180 degrees indicates a head-on conflict and an angle of 0 degrees indicates an in-trail conflict. Again, the data for this figure are for all conflicts where 


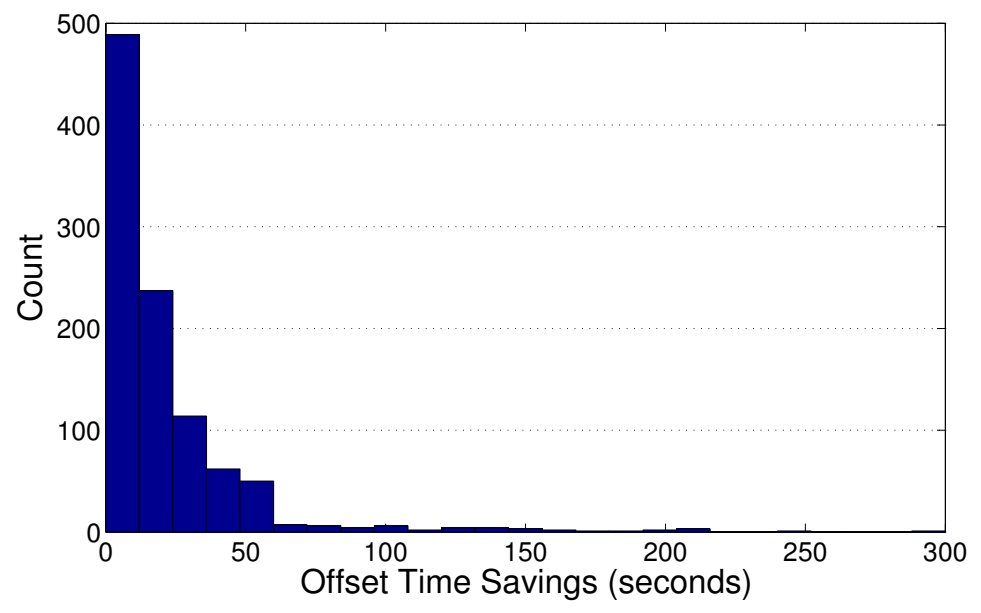

Figure 10. A histogram of the time savings for offset resolutions for the 2014 demand set and $50 \%$ path-stretch extension.

there were both a successful offset and a successful path-stretch resolution avialable. For each case, the lowest delay resolution type and the conflict angle were identified. Over $50 \%$ of the in-trail conflicts are more efficiently handled by an offset resolution, while only around $30 \%$ of the head-on conflicts are more efficiently handled by offsets. Thus, it can be seen from the figure that path-stretch resolutions seem to be more appropriate for higher encounter angles (i.e. head-on collisions), while offset resolutions are more efficient for in-trail type conflicts.

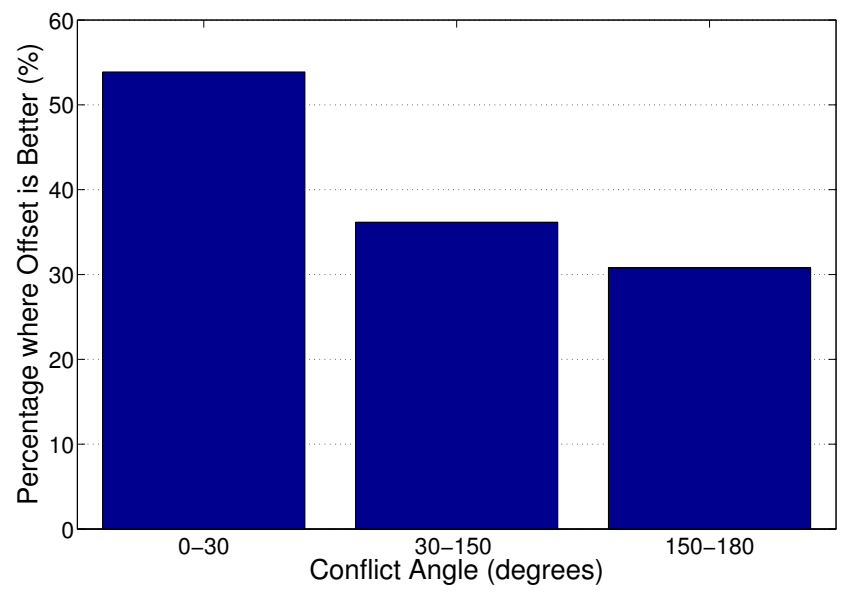

Figure 11. The percentage of cases for each conflict angle bin where the offset resolution was more efficient than the path-stretch resolution.

\section{Conclusions}

This paper presented the results of two studies aimed at understanding the effects and properties of parallel-offset conflict resolution maneuvers in the NAS. In the first study, the AAC Autoresolver was calibrated to approximate the performance of an air-traffic controller. These simulations indicated that facilitating parallel-offset conflict resolution maneuvers may lead to reduced system delay and improved system efficiency. Further, the data analysis indicated that path-stretch resolutions were the resolution type most likely to be replaced operationally by these offset resolutions.

A related, second study explored further the relationship between offset resolutions and path-stretch 
resolutions. The efficiency of the path-stretch resolution was used as an independent parameter, and the time savings and relative efficiency of path-stretch resolutions versus offset resolutions were presented. An analysis of the conflict properties revealed that offset resolutions may be more efficient in handling in-trail type resolutions than path-stretch resolutions.

Overall, the results presented here indicate that offset resolutions may be a viable method to increase system efficiency, especially as a replacement for horizontal vectoring and in-trail conflicts. Further studies can explore the possible additional benefits of offset resolutions in reducing controller workload and in increasing the accuracy of ground automation and decision support tools.

\section{References}

\footnotetext{
${ }^{1}$ Herndon, A. A., DeArmon, J. S., and Spelman, J., "Use of Lateral/Parallel FMS Procedures and Implementation Issues," Digital Avionics Systems Conference, 2004.

${ }^{2}$ Mondoloni, S., "Aircraft Trajectory Prediction Errors: Including a Summary of Error Sources and Data," Tech. rep., FAA EUROCONTROL Action Plan 16: Common Trajectory Prediction Capabilities, 2006.

${ }^{3}$ Paglione, M., Bayraktutar, I., McDonald, G., and Bronsvoort, J., "Lateral Intent Error's Impact on Aircraft Prediction," Proceedings of the 8th USA/Europe ATM 2009 RED Seminar, 2009.

${ }^{4}$ Meyn, L., Windhorst, R., Roth, K., Drei, D. V., Kubat, G., Manikonda, V., Roney, S., Hunter, G., and Couluris, G., "Build 4 of the Airspace Concepts Evaluation System," AIAA Modeling and Simulation Technologies Conference and Exhibit,

${ }^{5}$ Erzberger, H., "Automated Conflict Resolution for Air Traffic Control," 25th Iternational Congress of the Aeronautical Sciences, 2006.

${ }^{6}$ Erzberger, H., Lauderdale, T. A., and Cheng, Y., "Automated Conflict Resolution, Arrival Management and Weather Avoidance for ATM," 27th Iternational Congress of the Aeronautical Sciences, Nice, France, 2010.

${ }^{7}$ Paglione, M. M., Santiago, C., Crowell, A., and Oaks, R. D., "Analysis of the Aircraft to Aircraft Conflict Properties in the National Airspace System," AIAA Guidance, Navigation, and Control Conference, 2008.

${ }^{8}$ Santiago, C. and Paglione, M., "FMS Parallel Route Offset and Reduced Separation ACES Fast-Time Simulation Study," DOT/FAA/CT-TN10/2, 2010.

${ }^{9}$ Lauderdale, T., "The Effects of Passive Coordination on Distributed Separation Assurance," 9th AIAA Aviation Technology, Integration, and Operations Conference, Hilton Head, SC, 2009.
} 2006. 